\title{
Erratum to: Electromagnetic dipole moments of charged baryons with bent crystals at the LHC
}

\author{
E. Bagli ${ }^{1}$, L. Bandiera ${ }^{1}$, G. Cavoto ${ }^{2}$, V. Guidi ${ }^{1}$, L. Henry ${ }^{3}$, D. Marangotto ${ }^{4}$, F. Martinez Vidal ${ }^{3}$, A. Mazzolari ${ }^{1}$,

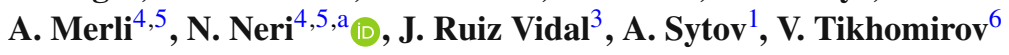 \\ ${ }^{1}$ INFN Sezione di Ferrara and Università di Ferrara, Ferrara, Italy \\ ${ }^{2}$ INFN Sezione di Roma and "Sapienza" Università di Roma, Roma, Italy \\ ${ }^{3}$ IFIC, Universitat de València-CSIC, Valencia, Spain \\ ${ }^{4}$ INFN Sezione di Milano and Universita' degli Studi di Milano, Milan, Italy \\ ${ }^{5}$ CERN, Geneva, Switzerland \\ ${ }^{6}$ Institute for Nuclear Problems, Belarusian State University, Minsk, Belarus
}

Received: 12 June 2020 / Accepted: 23 June 2020 / Published online: 28 July 2020

(C) The Author(s) 2020

\section{Erratum to: Eur. Phys. J. C (2017) 77:828 https://doi.org/10.1140/epjc/s10052-017-5400-x}

In this Erratum, an improved simulation of the channeling efficiency of protons and antiprotons as a function of the particle momentum is shown in Fig. 1 for different configurations of Si crystals bent along the (110) plane. Multiple scattering with nuclei and inner shell electrons was not properly taken into account and it has been corrected using the simulation code CRYSTAL [1], which is designed for simulations of trajectories of charged particles interacting with crystalline structures. The code solves the equation of motion for a charged particle interacting with the electric field generated by atomic strings or planes accounting for multiple scattering according to Refs. [2,3]. The code accounts for a wide variety of effects, namely multiple and single Coulomb scattering on nuclei and electrons, nuclear scattering, ionization losses, crystal geometry. and, for the case of leptons, emission of radiation. The model used in the code has been validated for electrons at sub- $\mathrm{GeV}$ energy, for electrons and positrons at $20 \mathrm{GeV}$, for electrons and positrons at $120 \mathrm{GeV}$, as well as for protons at few hundreds of $\mathrm{GeV}$ for crystals at room temperature.
The new distribution of the channeling efficiency for $7 \mathrm{~cm}$ long and $14 \mathrm{mrad}$ bent $\mathrm{Si}$ crystal is significantly different from the previous version, presenting a maximum around $400 \mathrm{GeV}$ and going to zero at lower energies. This result has no impact on the rest of the paper, since channeling efficiency for $\Lambda_{\mathrm{c}}^{+}$baryons is simulated using a parameterisation based on current theoretical description and channeling measurements, following Ref. [4].

In addition, a wrong plus and a wrong minus sign have been identified in Eqs. (5) and (7), respectively.

The corrected equations are reported below:

$$
\begin{aligned}
\boldsymbol{\Omega}_{\mathrm{EDM}} & =\frac{d \mu_{B}}{\hbar}\left(\mathbf{E}-\frac{\gamma}{\gamma+1}(\boldsymbol{\beta} \cdot \mathbf{E}) \boldsymbol{\beta}+\boldsymbol{\beta} \times \mathbf{B}\right), \\
s_{x} & \approx s_{0} \frac{d}{g-2}(1-\cos \Phi) .
\end{aligned}
$$

It is worth it to note that Eqs. (7) and (8) of the paper apply for up- and down-bending crystals, with the bending angle $\theta_{C}$ taken negative and positive, respectively. The equations are in agreement with Ref. [5] where an opposite sign convention is used for the bending angle $\theta_{C}$. The wrong sign errors have no impact on other results reported in the paper.

The original article can be found online at https://doi.org/10.1140/ epjc/s10052-017-5400-x.

\footnotetext{
a e-mail: nicola.neri@mi.infn.it (corresponding author)
} 


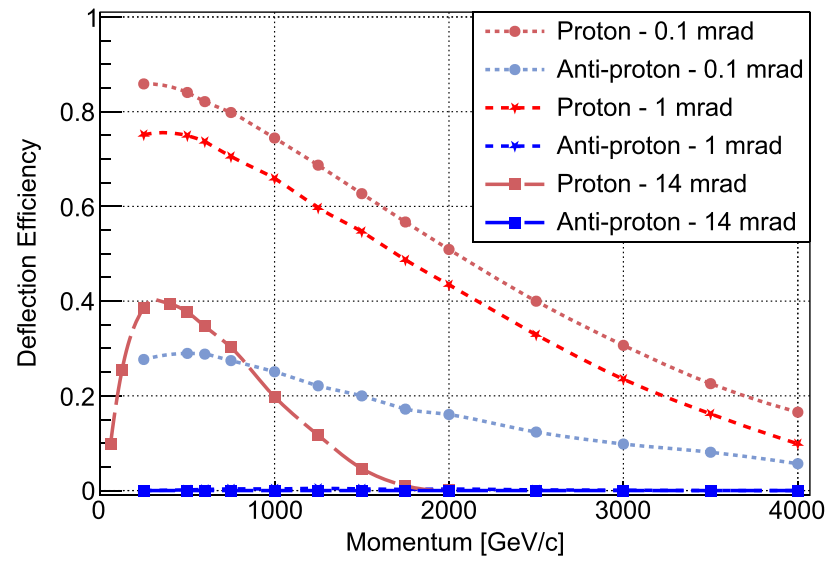

Fig. 1 Dependence of the channeling efficiency of protons and antiprotons with the particle momentum for $1 \mathrm{~mm}, 1 \mathrm{~cm}$ and $7 \mathrm{~cm}$ long $\mathrm{Si}$ crystals bent along the (110) plane by a $0.1 \mathrm{mrad}, 1 \mathrm{mrad}$, and $14 \mathrm{mrad}$ bending angle, respectively. The curves for the anti-proton interacting with the $1 \mathrm{~cm}$ and $7 \mathrm{~cm}$ long Si crystals are superimposed

Acknowledgements We acknowledge support from INFN (Italy), MINECO and GVA (Spain), the ERC Consolidator Grant CRYSBEAM G.A. 615089, and the ERC Consolidator Grant SELDOM G.A. 771642. In addition, we acknowledge the CINECA award under the ISCRA initiative for the availability of high-performance computing resources and support.

Data Availability Statement This manuscript has no associated data or the data will not be deposited. [Authors' comment: The relevant data are presented in Figure 1 of the erratum.]

Open Access This article is licensed under a Creative Commons Attribution 4.0 International License, which permits use, sharing, adaptation, distribution and reproduction in any medium or format, as long as you give appropriate credit to the original author(s) and the source, provide a link to the Creative Commons licence, and indicate if changes were made. The images or other third party material in this article are included in the article's Creative Commons licence, unless indicated otherwise in a credit line to the material. If material is not included in the article's Creative Commons licence and your intended use is not permitted by statutory regulation or exceeds the permitted use, you will need to obtain permission directly from the copyright holder. To view a copy of this licence, visit http://creativecomm ons.org/licenses/by/4.0/.

Funded by SCOAP ${ }^{3}$.

\section{References}

1. A. Sytov, V. Tikhomirov, Nucl. Instrum. Methods B 355, 383 (2015). https://doi.org/10.1016/j.nimb.2015.02.042

2. A.I. Sytov, V.V. Tikhomirov, L. Bandiera, Phys. Rev. Accel. Beams 22, 064601 (2019). https://doi.org/10.1103/PhysRevAccelBeams. 22.064601

3. A. Mazzolari et al., Eur. Phys. J. C 80(1), 63 (2020). https://doi.org/ 10.1140/epjc/s10052-019-7586-6

4. V.M. Biryukov et al., Crystal Channeling and Its Application at High-Energy Accelerators (Springer, Berlin, Heidelberg, 1997)

5. J. Fu, M.A. Giorgi, L. Henry, D. Marangotto, F.M. Vidal, A. Merli, N. Neri, J.R. Vidal, Phys. Rev. Lett. 123(1), 011801 (2019). https:// doi.org/10.1103/PhysRevLett.123.011801 